\title{
A comparison of two theoretical methods of the materials transportation in the vibrating screen machines and improve the significance of their experimental results
}

\author{
Jamil Sami Haddad ${ }^{1}$, Waleed Momani ${ }^{2}$ \\ I (Mechanical Engineering Department, Faculty of Engineering Technology / Al Balqa Applied University, \\ Jordan) \\ ${ }^{2}$ (Mechanical Engineering Department, Faculty of Engineering Technology / Al Balqa Applied University, \\ Jordan)
}

\begin{abstract}
:
Problem statement: This study was made to increase productivity of separating process in many industries, to achieve the required aim, understanding the relationship between designed arithmetic methods on two and three coordinate planes to choose the parameters of the separating machine and computing additional factors including characteristics of materials and type of screen.

Approach: The design of these types of separating machines did not include the addressed factors during the design; the study of these new factors will provide better understanding of the process and increase the abilities for improvement. Study made to define the right parameters of the operating surface, amplitude and vibration frequency that provide necessary productivity, efficient parameters of material.

Results: The improvement included the machine design which consisted of screen; the screen design includes size, angle of inclination and taking into consideration the effect of the vibration trajectory. After applying primary data and the standard computation, the dynamic parameters are set and then the geometric parameters. According to the mathematical computation the design was made and then the verifying of the elements of speed of material motion. These mathematical equations can solve the problem of low productivity and high power consumption if implemented correctly and taking into consideration the dynamic and geometric elements of each case.

Conclusion: The results of this study can be implemented in many fields of the industry where separation machine were used, the application of the mathematical relations can improve the revenue by increasing productivity and lowering power consumption for industry. For further study, the effect of wavy surface on the movement of particles will be advised.
\end{abstract}

Keywords: Amplitude, Conveyor, Dynamic, frequency, Screen, Vibration.

\section{Introduction}

Vibratory classification of rock mass by size is one of the main operations in mineral processing. This opera-tion has become widespread in various industries such as mining, metallurgy, chemical industry, construction and food industry. In all these enterprises is exploited over a thousand different frame sizes of vibrating screens differing both structurally and modes of their work.

Further improvement of the screen's construction is a very urgent task, especially when the classification of small and thin size rock mass. In addition, any special requirements are demanded for new constructions of vibrating screens in today's market of mining machinery, such as high technological parameters, low energy and metal, as well as high wear resistance. In such a framework the production of the screens is put in the situation of rising requirements to the quality of the produced raw materials and intermediate products, increased processing of low-grade raw materials, as well as rising energy costs and consumables.

Significant scientific and practical interests are the investigations aimed at exploring the possibilities of using in order to increase the technological performance of the screening with a spatial excitation of the sieving surface. Use of vibrating unbalanced self-synchronizing drive allows creating a rather simple construction of vibrator having numerous advantages: low power consumption, the complex nature of movement of the working body, contributing to the intensification of the process of sieve self-cleaning in the classification of difficult screening materials by using as a vibrating drive easy to use motor-vibrators.

Important and necessary condition for the effectiveness of the classification process is a sufficient dislodgement of a sieving material. Due to the excitation of the vibratory screening surface can reduce the path length of the particles within the material layer to the sieve surface, given the location of their contact, the force of adhesion and the internal friction between the particles. Thus the process of vibratory screening includes 
continuous momentum exchange between the sieve and feedstock (recyclable raw materials). Thus, the nature of the vibration sieve is of great importance in determining the discrete values of the transmitted energy pulse of a separate material particle $[1,3]$.

\section{Methodology And Materials}

The main requirement for the dynamic scheme of the screen is to provide the necessary, chosen from the conditions optimal behavior of a technological process one of the masses of law oscillations, which is its working body. While the dynamic scheme should provide the required level and the basic operational characteristics of vibrating screen: stability, balance and amplification factor of the driving force.

On horizontal and slightly inclined screens to enhance their technological performance can answer the implementation of the authors' proposed layout of inertial self-synchronizing vibration exciters (Fig. 2.1. (a)), which creates a spatial variation of the screen box [5], and (Fig. 2.1 (b)) were just changed the direction of force $\mathrm{P}_{0}$ [1]. The scheme consists of two unbalanced exciters with a rotating acceleration vector, located on the sides at a distance from the center of system's mass perpendicular to the transverse axis of the car box. The planes of unbalanced masses rotation are inclined in opposite directions at a certain angle $\beta$ to the plane of the sieving surface.

While counter-rotating shaft of exciters' the unbalanced masses are rotated to a corresponding phase angle $\left(\varphi_{1}=\omega \cdot t\right.$ and $\left.\varphi_{2}=\pi-\omega \cdot t\right)$ and with equality of their unbalanced masses (m1 = m2) develop equal centrifugal force of inertia $\mathrm{P}_{0}[1,2,6]$.

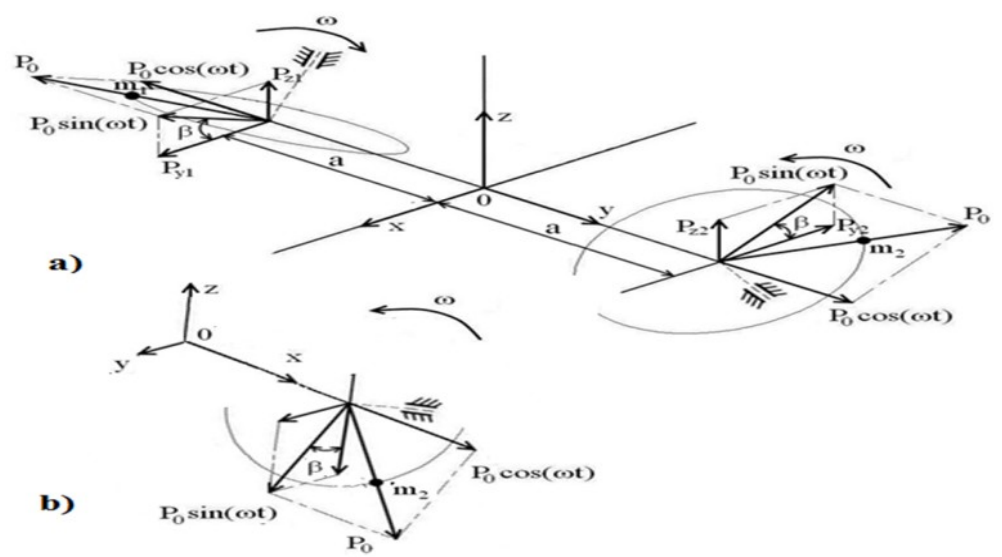

Fig. 2.1 - Scheme of screening forces distribution with two unbalance exciters

The sum of the projections of all forces on each of the three coordinate axes $(0 x, 0 y, 0 z)$ and the sum of their moments relative to the center (point 0 ) lying in the plane of the forces, represented by systems of equations:

$\sum P_{x}=P_{0} \sin (\omega t) \sin \beta+P_{0} \sin (\pi-\omega t) \sin (-\beta)=0$

$\sum P_{y}=P_{0} \cos (\omega t)+P_{0} \cos (\pi-\omega t)=0$

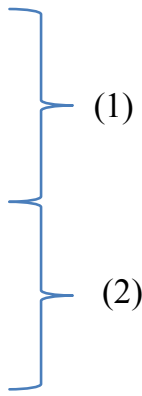

$\sum P_{z}=P_{0} \sin (\omega t) \cos \beta+P_{0} \sin (\pi-\omega t) \cos (-\beta)=2 P_{0} \sin (\omega t) \cos \beta$

$\sum M_{x}=P_{0} \sin (\omega t) \cos (\beta) a-P_{0} \sin (\pi-\omega t) \cos (-\beta) a=0$

$\sum M_{y}=0$

$\sum M_{z}=P_{0} \sin (\omega t) \sin (\beta) a+P_{0} \sin (\pi-\omega t) \sin (-\beta) a=2 P_{0} \sin (\omega t) \sin (\beta) a$

To simplify the analysis of the basic motion laws of screening surface we will accept assumption: screen box is absolutely a rigid body. Moment of couple of force $\left(2 \cdot P_{0} \cdot \sin (\omega \cdot t) \cdot \sin (\beta) \cdot a\right)$ equals by the module to the product to the module one of the forces on the shoulder of the pair $(2 \cdot a)$ [7].

From systems of equations (1) and (2) follow that while working on the vibratory drive the screen box acts harmonic perturbing force $\left(2 \cdot P_{0} \cdot \sin (\omega \cdot t) \cdot \cos (\beta)\right)$ and the moment ( $\left.2 \cdot P_{0} \cdot \sin (\omega \cdot t) \cdot \sin (\beta) \cdot a\right)$ directed, accordingly, along and around the axis $0 z$ and creating translational alternating vibrations of the screening along the vertical axis [1]. 


\section{The Basic Theoretical Backgrounds of the trajectory of material motion in the tossing mode}

Established character of the variations allows determining the behavior of the material particles (trajectory and speed) on the screening surface of the vibrating screen, that in the future will allow developing a new design of the machine when implementing the principle of resource saving of its construction.

The fig. 3.2 below, shows the calculation scheme of vibration displacement of a material particle by the mass $\mathrm{m}$ (using the original coordinates $\mathrm{X}: \mathrm{Y}$ ) at a certain angle $\alpha$, taking into consideration the effect of the air current $\mathrm{P}$ with a certain angle $\gamma[3,4]$.

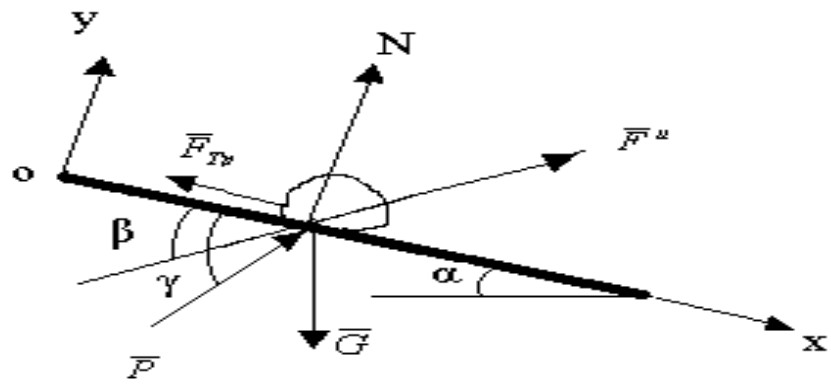

Fig. 3.2 - Calculation scheme of vibration displacement of a material particle on sieving surface of the screening using the original coordinates $\mathrm{X}$ : $\mathrm{Y}$

$$
\begin{aligned}
& \mathrm{m} \ddot{x}=F^{u} \cos \beta+\mathrm{G} \sin \alpha+\mathrm{P} \cos \gamma-F_{T p} \\
& \mathrm{~m} \ddot{y}=F^{u} \sin \beta-\mathrm{G} \cos \alpha+\mathrm{P} \sin \gamma-\mathrm{N}
\end{aligned}
$$

Calculation scheme of vibration displacement of a material particle by the mass $m$ (using the original coordinates $\mathrm{X}: \mathrm{Y}: \mathrm{Z}$ ) on sieving surface of the screening with spatial variations of the box, inclined to the horizon at a certain angle $\alpha$, shown in Fig. 3.4, [1,2].

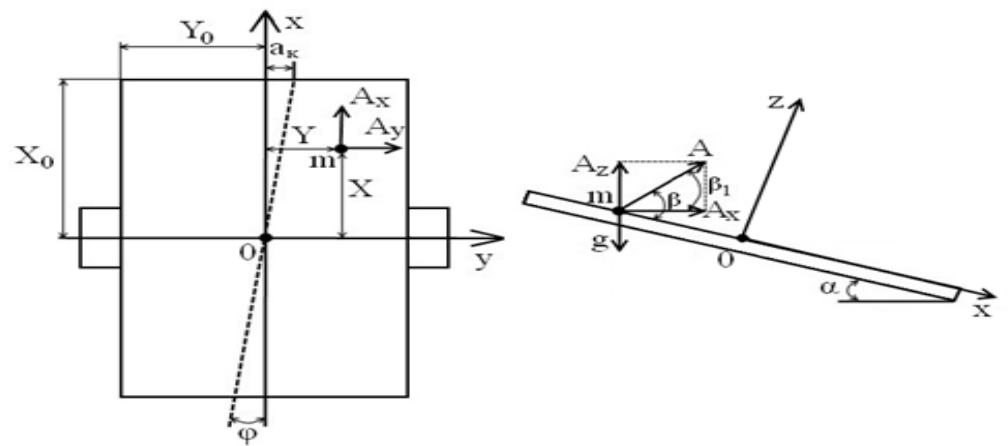

Fig. 3.4 - Calculation scheme of vibration displacement of a material particle on sieving surface of the screening using the original coordinates $\mathrm{X}: \mathrm{Y}: \mathrm{Z}$

Particle motion of the material in three coordinate planes is described by the system of equations $[2,7]$ :

$$
\left\{\begin{array}{l}
m \cdot \ddot{x}=m \cdot A_{x} \cdot \omega^{2} \cdot \cos \beta \cdot \sin \omega \cdot t+m \cdot g \cdot \sin \alpha ; \\
m \cdot \ddot{y}=m \cdot A_{y} \cdot \omega^{2} \cdot \sin \omega \cdot t ; \\
m \cdot \ddot{z}=m \cdot A_{z} \cdot \omega^{2} \cdot \sin \beta \cdot \sin \omega \cdot t-m \cdot g \cdot \cos \alpha,
\end{array}\right.
$$

where $A_{x}=Y \cdot a_{\varphi}, A_{y}=X \cdot a_{\varphi}, A_{z}$-is the amplitude of variations on the coordinate axes, мм; $\varphi$ - is an angle of plane's rotation of the sieve relative to the axis $x$, grad ..

Particle motion of the material in tow coordinate planes is described by author [7] taking into consideration the effect of bending oscillations on the motion of the material in the vibro-drying machines [7]:

Rotary fluctuations amplitude of the box:

$$
a_{\varphi}=\frac{a_{k}}{X_{0}}
$$

where $a_{k}$ - is the amplitude of horizontal fluctuation on the contour of the box, MM; $X_{0}$ - is a distance from the center of the box to its discharge end, $\mathrm{mm}$. 
Coefficient of the vibro- displacement mode at the particles location point where the:

$$
\tilde{A}=\frac{A \cdot \omega^{2} \cdot \sin \beta}{g \cdot \cos \alpha}
$$

where $A=\sqrt{A_{z}^{2}+A_{x}^{2}}$ - is a resulting amplitude of variations of the screen box, mm; $\omega$ - is a frequency of variation, $c^{-1} ; \beta=\beta_{1}+\alpha ; \beta_{1}=\arctan \frac{A_{z}}{A_{x}}-$ vibration direction angle to the horizon, deg.; $\alpha$ inclination angle of the sieving surface, deg.

Equation (4) and phase angle of the separation of a material particle from the surface of the sieve $\psi_{o}$ are related by $[3,4,6]$ :

$$
\psi_{o}=\arcsin \frac{1}{\tilde{A}}
$$

From the system of equations (3) we will define the law of motion of a particle with tossing relative to the coordinate's $z$, entering as a variable phase:

$$
\begin{gathered}
\ddot{z}=A_{z} \cdot \omega^{2} \cdot \sin \beta \cdot \sin \psi-g \cdot \cos \alpha \\
\dot{z}=-A_{z} \cdot \omega \cdot \sin \beta \cdot \cos \psi-g \cdot \cos \alpha \cdot \frac{\psi}{\omega}+C_{1} \\
z=-A_{z} \cdot \sin \beta \cdot \sin \psi-g \cdot \cos \alpha \cdot \frac{\psi^{2}}{2 \cdot \omega^{2}}+C_{1} \cdot \frac{\psi}{\omega}+C_{2}
\end{gathered}
$$

From the conditions of material separation from the working surface (initial conditions) : $\dot{z}=0 ; z=\mathrm{O}$ by $t=t_{o}\left(\psi=\psi_{o}\right)$ for $t=t_{o}\left(\psi=\psi_{o}\right)$ we define $C_{1}$ and $C_{2}(5)$ and (6):

$$
\begin{aligned}
& C_{1}=A_{z} \cdot \omega \cdot \sin \beta \cdot \cos \psi_{o}+g \cdot \cos \alpha \cdot \frac{\psi_{o}}{\omega} ; \\
& C_{2}=A_{z} \cdot \sin \beta \cdot \sin \psi_{o}+g \cdot \cos \alpha \cdot \frac{\psi_{o}^{2}}{2 \cdot \omega^{2}}-C_{1} \cdot \frac{\psi_{o}}{\omega}=A_{z} \cdot \sin \beta \cdot \sin \psi_{o}+ \\
& +g \cdot \cos \alpha \cdot \frac{\psi_{o}^{2}}{2 \cdot \omega^{2}}-A_{z} \cdot \sin \beta \cdot \cos \psi_{o} \cdot \psi_{o}-g \cdot \cos \alpha \cdot \frac{\psi_{o}^{2}}{\omega^{2}}
\end{aligned}
$$

Substituting the values of constant coefficients (7) and (8) in equation (6), we define $z$ :

$$
\begin{aligned}
& z=-A_{z} \cdot \sin \beta \cdot \sin \psi-g \cdot \cos \alpha \cdot \frac{\psi^{2}}{2 \cdot \omega^{2}}+A_{z} \cdot \omega \cdot \sin \beta \cdot \cos \psi_{o} \cdot \psi+g \cdot \cos \alpha \cdot \frac{\psi_{o} \cdot \psi}{\omega^{2}}+ \\
& +A_{z} \cdot \sin \beta \cdot \sin \psi_{o}+g \cdot \cos \alpha \cdot \frac{\psi_{o}^{2}}{2 \cdot \omega^{2}}-A_{z} \cdot \sin \beta \cdot \cos \psi_{o} \cdot \psi_{o}-g \cdot \cos \alpha \cdot \frac{\psi_{o}^{2}}{\omega^{2}}
\end{aligned}
$$

Taking into consideration (4), the equation becomes:

$$
z=A_{z} \cdot \sin \beta \cdot\left[\sin \psi_{o}-\sin \psi+\cos \psi_{o} \cdot\left(\psi-\psi_{o}\right)-\frac{\left(\psi-\psi_{o}\right)^{2}}{2 \cdot \widetilde{A}}\right] .
$$

Determine the coordinates of $\Delta \mathrm{z}$ meeting of a material particle with the plane of the screening working body (at $\psi=\psi_{\hat{a}}$ ):

$$
\Delta z=A_{z} \cdot \sin \beta \cdot\left[\sin \psi_{o}-\sin \psi_{\hat{a}}+\cos \psi_{o} \cdot\left(\psi_{\hat{a}}-\psi_{o}\right)-\frac{\left(\psi_{\hat{a}}-\psi_{o}\right)^{2}}{2 \cdot \tilde{A}}\right]
$$
conditions:

From equation (9) we will define phasic meeting angle of the particle with the surface $\psi_{\mathrm{v}}$ under boundary

$$
\sin \psi_{o}-\sin \psi_{\hat{a}}+\cos \psi_{o} \cdot\left(\psi_{\hat{a}}-\psi_{o}\right)-\frac{\left(\psi_{\hat{a}}-\psi_{o}\right)^{2}}{2 \cdot \tilde{A}}=0
$$

Transcendental equation. Considering that the particle moves with continuous tossing we assume that $\psi_{\hat{a}}$ is close to $2 \pi$. In this case $\psi_{\hat{a}}=2 \pi+\Delta \psi_{\hat{a}}$. Expression (10) takes the form: 
$\sin \psi_{0}-\sin \left(2 \pi+\Delta \psi_{\hat{a}}\right)-\frac{\left(2 \pi+\Delta \psi_{\hat{a}}-\psi_{0}\right)^{2}}{2 \cdot \tilde{A}}+\cos \psi_{0} \cdot\left(2 \pi+\Delta \psi_{\hat{a}}-\psi_{0}\right)=0$

Hence, considering that for small, we obtain:

Where:

$$
\begin{gathered}
\Delta \psi_{\hat{a}}=-\frac{1}{2} \cdot b+\sqrt{\left(\frac{1}{2} \cdot b\right)^{2}-c} \\
b=4 \pi-2 \cdot \psi_{0}+2 \cdot \tilde{A} \cdot\left(1-\cos \psi_{0}\right) \\
c=4 \pi^{2}+\psi_{0}^{2}-4 \pi \cdot \psi_{0}-2 \cdot \tilde{A} \cdot\left[\sin \psi_{0}+\left(2 \pi-\psi_{0}\right) \cdot \cos \psi_{0}\right]
\end{gathered}
$$

Similarly we will define coordinates for longitudinal $x$ and transverse $y$ displacement of a material particle. As a result of the calculation obtained the following equation:

$$
\begin{aligned}
& x=-A_{x} \cos \beta\left[\sin \psi_{0}-\sin \psi+\cos \psi_{0}\left(\psi-\psi_{0}\right)+\frac{\tan \alpha \tan \beta\left(\psi-\psi_{0}\right)^{2}}{2 \AA}\right] \\
& \left.y=A_{y} \cdot\left[\cos \psi_{o} \cdot\left(\psi-\psi_{o}\right)-\sin \psi+\sin \psi_{o}\right)\right]
\end{aligned}
$$

Coordinates of meeting of a material particle with the sieve plane (at $\psi=\psi_{\hat{a}}$ ):

$$
\begin{aligned}
& \Delta x=-A_{x} \cos \beta\left[\sin \psi_{0}-\sin \psi_{\hat{a}}+\cos \psi_{0}\left(\psi_{\hat{a}}-\psi_{0}\right)+\frac{\tan \alpha \tan \beta\left(\psi_{\hat{a}}-\psi_{0}\right)^{2}}{2 \AA}\right] \\
& \left.\Delta y=A_{y} \cdot\left[\cos \psi_{o} \cdot\left(\psi_{\hat{a}}-\psi_{o}\right)-\sin \psi_{\hat{a}}+\sin \psi_{o}\right)\right]
\end{aligned}
$$

Average speed vibro-displacement particles:

$$
v_{\tilde{n} \circlearrowright}=\frac{\Delta x \cdot \omega}{2 \pi} .
$$

Here, it is possible to compare the equations $(12,13)$ with work $[3,4]$ where the author found in the same way the average speed vibro-displacement particles on the surface. The value of particle displacement over one period of oscillation of the operating member is defined:

$$
\Delta S=A \cos \beta\left[\sin \psi_{0}-\sin \psi_{B}+\cos \psi_{0}\left(\psi_{B}-\psi_{0}\right)+\frac{1}{2} Q\left(\psi_{B}-\psi_{0}\right)^{2}\right]
$$

and average velocity of vibro-transporting.

$$
v_{c p}=\frac{\Delta S \omega}{2 \pi} .
$$

The obtained equations allow to simulate trajectory of particles' movement on the working body $(700 \times$ $1300 \mathrm{~mm})$ of the screening by the software tools «Mathcad». The authors [1,2] had working body $(500 \times 1500$ $\mathrm{mm}$ ). Input parameters: $A_{x}=1 \mathrm{~mm}, A_{y}=1,5 \mathrm{~mm}, A_{z}=2 \mathrm{~mm}, \omega=25 \mathrm{~Hz}, \beta=45$ deg., $\alpha=15$ deg.

Represented analytical expressions for determining the particle's trajectory of rock mass on the sieving surface of the screening with adjustable spatial trajectory of variations allow investigating the path and speed of movement of the material's particles of different masses and density in the mode of tossing [2].

\section{Constructive realization of vibrating screen with the transverse fluctuations of The working body and its testing}

To confirm the effectiveness of the developed scheme of the vibratory drive were carried out comparative tests of the screening technological parameters with circular, directional and spatial trajectory of variations, most recent of which are implemented by the proposed kinematics. Experimental stand-screening, shown on the Fig. 4.4, consists of set on the support frame 6 through elastic dampers 5 of a box 3 with a sieving surface1. The mounted vibratory drive 4 and rubber 2 is used for creating spatial and circular oscillations of the box and during the installation of the vibration exciters 4 at the same angle to the horizon ( $45 \mathrm{deg}$.) obtained directed variations. As shakers in all the schemes were used vibration exciters $\left(P_{\text {nom }}=0.12 \mathrm{~kW}, \omega_{\text {nom }}=1500\right.$ $\mathrm{r} / \mathrm{min}$ ). For all the investigated schemes vibration perturbation design parameters were constant, and the identity of regime parameters were provided equal to the power consumption of $0.1 \mathrm{~kW} \pm 5 \%$ by changing the installation angle of the unbalanced masses (static moment) on the shaft of the vibration exciters[1,2]. 


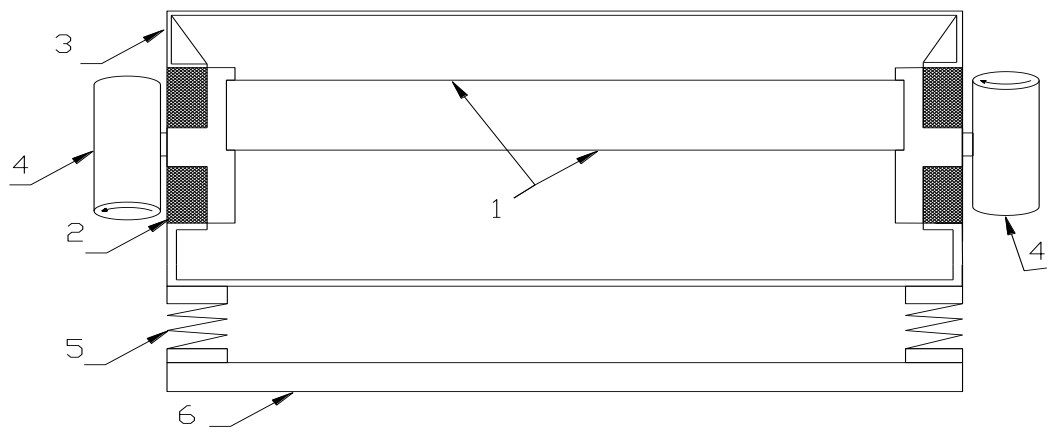

1 - sieving surface; 2 - internal vibration (rubber); 3 - duct (box); 4 - vibratory drive; 5 - dampers (springs); 6 - frame

Figure 4.4 - The construction of an experimental stand-screening

Comparative tests of technological parameters of the process - performance of the screening on the initial nutrition and efficiency of the classification process with spatial $\left(\mathrm{Q}_{\mathrm{s}}\right.$ and $\left.\mathrm{E}_{\mathrm{s}}\right)$, circular $\left(\mathrm{Q}_{c}\right.$ and $\left.\mathrm{E}_{\mathrm{c}}\right)$ and directional $\left(\mathrm{Q}_{\mathrm{d}}\right.$ and $\left.\mathrm{E}_{\mathrm{d}}\right)$ trajectories variations of the working body undertook by the separation of tuff (class of $10 \mathrm{~mm}, \mathrm{~g} / \mathrm{cm}^{3} \rho=15$ ) weighing $50 \mathrm{~kg}$., as such as in the work [1].

Fig. 4.5, shows the dependence of technological parameters from vibration exciter's shaft rotation frequency $\omega(\mathrm{r} / \mathrm{min})$, which shows that the dependence of all three types of trajectories of variations remains. It should be noted that the screening efficiency for the entire range $\omega$ while working of the screening with the proposed scheme dynamic for $7-13 \%$ is higher greater with $2-3 \%$ of performance by the initial nutrition than with the scheme of a rotational trajectory, but below $4.3 \%$ than the scheme with directional variations[1]. The curve 7 and 8 shows more efficiency and productivity when same cross sectional area but greater in width and less in length.

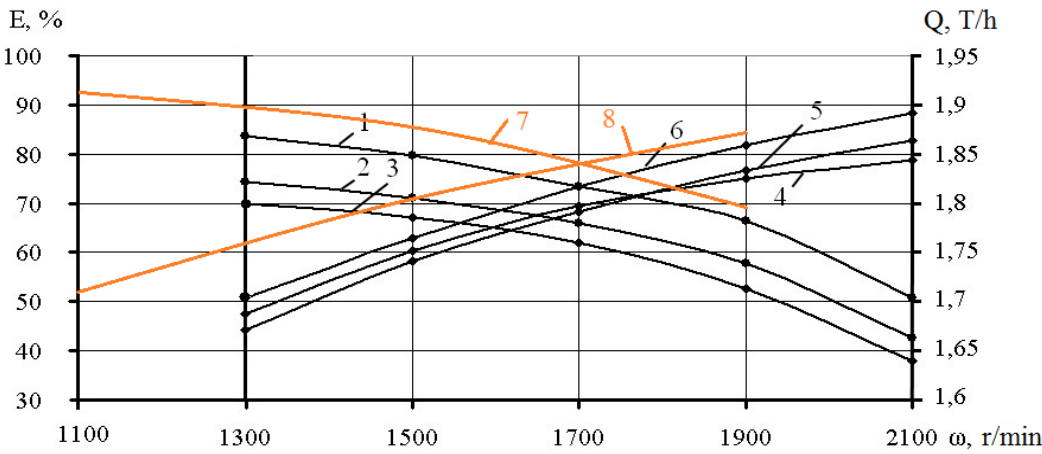

a) with cross sectional area $(500 \times 1500 \mathrm{~mm}): 1-\mathrm{E}_{\mathrm{s}}(\omega) ; 2-\mathrm{E}_{\mathrm{c}}(\omega) ; 3-\mathrm{E}_{\mathrm{d}}(\omega) ; 4-\mathrm{Q}_{\mathrm{c}}(\omega) ; 5-\mathrm{Q}_{\mathrm{s}}(\omega) ; 6-$

$\left.\mathrm{Q}_{\mathrm{d}}(\omega) . \mathrm{b}\right)$ with cross sectional area $(700 \times 1300 \mathrm{~mm}): 7-\mathrm{E}_{\mathrm{s}}(\omega) ; 8-\mathrm{Q}_{\mathrm{d}}(\omega)$;

Fig. 4.5 - Dependence of technological parameters from vibration exciter's shaft rotation frequency $\omega$

Experimental dependence of technological parameters from the angle of inclination of the sieving surface (Fig. 4.6) showed that the efficiency of the screening while the spatial excitation of the sieve is higher than the other two. Thus, the performance of the screening with the angle exceeding 15 degrees for exceeds $2 \%$ than given indicator of work in comparison with the scheme of linear variations and for $4 \%$ - in comparison with circular. As for the curve 7, we find a relatively high efficiency whenever a few angle, but compared to the previous works, we find a small decrease in the amount of production. 


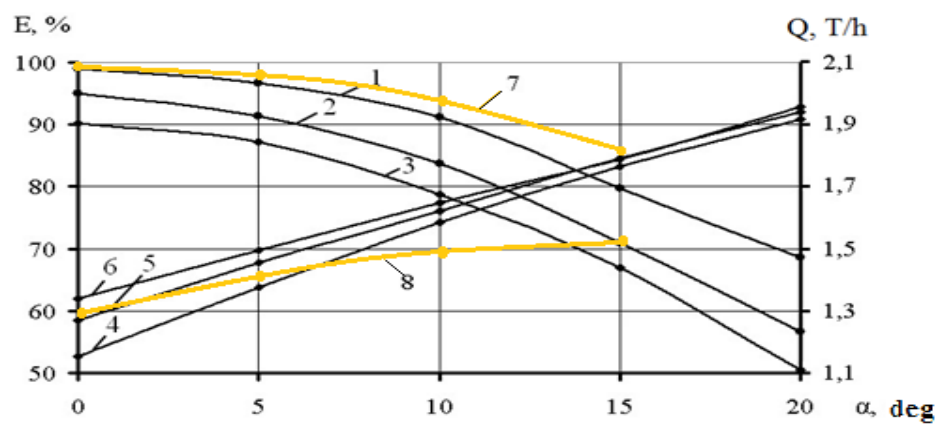

a) with cross sectional area $(500 \times 1500 \mathrm{~mm}): 1-\mathrm{E}_{\mathrm{s}}(\omega) ; 2-\mathrm{E}_{\mathrm{c}}(\omega) ; 3-\mathrm{E}_{\mathrm{d}}(\omega) ; 4-\mathrm{Q}_{\mathrm{c}}(\omega) ; 5-\mathrm{Q}_{\mathrm{s}}(\omega) ; 6-$

$\mathrm{Q}_{\mathrm{d}}(\omega)$. b) with cross sectional area $(700 \times 1300 \mathrm{~mm}): 7-\mathrm{E}_{\mathrm{s}}(\omega) ; 8-\mathrm{Q}_{\mathrm{d}}(\omega)$;

Fig. 4.6 - The dependence of technological parameters from the angle of inclination of the sieving surface $\alpha$

\section{Conclusion}

The proposed design of dampers and choose the dynamic scheme of the screening vibratory drive allows to create alternating translational variations of the screen box along its vertical axis. The choice of suitable sectional area with the small increase in the width of the surface works to increase the efficiency of the classification process. Spatial variations of the working body can intensify the movement of the material on the screening surface in the longitudinal and transverse directions. Thus increases the technological parameters of the classification process due to the movement of the material in a weaving path in which increases the length of the path of the rock mass on the sieving surface. Additional to transverse the longitudinal variations of the box also enable more efficient use of space by the sieve width.

\section{References}

[1] V.P. Franchuk, V.P. Naduty, and P.V. Levchenko, Increasing technological parameters bulk material by improving the dynamic schema, Proceedings of the National Technical University. Series: Chemistry, Chemical Engineering and Environment, 59 (965), 2012, 132-138.

[2] V.P. Franchuk, V.P. Naduty, and P.V. Levchenko, Determination of the trajectory of motion of the material, taking into account the transverse oscillations vibrating screen, Proceedings of the National Technical University. Series: Chemistry, Chemical Engineering and Environment, 57 (965), 2013, 142-148

[3] J. S. Haddad, Basic Theoretical Backgrounds of the Engineering Design Procedure of a Drying Plant, American J. of Eng. and Applied Sciences 2(2), 2009, 466-470.

[4] F. Al-Quran, J. S. Haddad, M. Dweirj. Calculation Procedure to Choose the Design Parameters of Drying Machines, Springer, J. of Eng. Thermophysics, 4(18), 2009, 315-320

[5] V.P. Naduty, P.V. Levchenko, Vibrating screen, Patent for useful model, 77403 UA, MPK8 B 07 B 1/40 (2006.01).National Academy of Sciences, Bull. 3, 11.02.2013, - 4

[6] V.N. Chelome, Vibrations in Technology, Vibrating processes and machines (A handbook In 6 vols. Mechanical Engineering, E.E. Lavendel, T. 4 / 1981, 509).

[7] N.N. Buchholts The main course of theoretical mechanics. Part 1 kinematics, statics, dynamics of a material point (8th ed. - M .: Science, 1969, 468) 